\title{
Imagem corporal de mulheres com câncer de mama: uma revisão sistemática da literatura
}

\author{
Body image of women with breast cancer: \\ a systematic review of literature
}

Daniela Barsotti Santos ${ }^{1}$ Elisabeth Meloni Vieira ${ }^{2}$

\footnotetext{
${ }^{1}$ Escola de Enfermagem de Ribeirão Preto,

Universidade de São Paulo. Av. Bandeirantes 3900, Monte Alegre. 14040-902 Ribeirão Preto SP. danibarsotti@yahoo.com.br ${ }^{2}$ Departamento de Medicina Social, Faculdade de Medicina de Ribeirão Preto, Universidade de São Paulo.
}

\begin{abstract}
Women experience a major process of reshaping their body image when they deal with breast cancer. This article seeks to understand the relationship that breast cancer and its treatment have in the process of (re)construction of a woman's body image. The ultimate objective is to promote knowledge to train health professionals to become more aware of a woman's quality of life. A systematic review of the literature of scientific articles published between 2004 and 2009 available in three scientific databases was conducted and a total of 56 articles were reviewed and grouped into four thematic categories. There is a pressing need for further studies on the socio-cultural characteristics of women with breast cancer, the differences of (re)construction of body image of young and older women, and Brazilian publications about the personal experience and socio-cultural context of women with breast cancer.
\end{abstract}

Key words Breast cancer, Body image, Psychological adjustment
Resumo A mulher passa por um importante processo de reformulação da imagem corporal quando lida com o câncer de mama. Este artigo objetiva a compreensão da relação que o câncer de mama e seus tratamentos têm no processo de (re)elaboração da imagem corporal das mulheres, visando assim ao fomento de subsídios para a formação e a capacitação de profissionais de saúde mais atentos à promoção da qualidade de vida delas. Foi realizada uma revisão sistemática da literatura de artigos científicos publicados entre 2004 e 2009 disponíveis em três bases de dado; 56 artigos foram revisados e agrupados em quatro categorias. Destacamos a necessidade de mais estudos que contemplem características socioculturais de mulheres com câncer de mama, sobre diferenças na (re)elaboração da imagem corporal de mulheres jovens e de mulheres mais velhas, e de publicações brasileiras sobre a experiência pessoal e aspectos socioculturais específicos de mulheres com câncer de mama.

Palavras-chave Neoplasias da mama, Imagem corporal, Ajustamento psicológico 


\section{Introdução}

O câncer de mama continua sendo uma das principais causas de morte de mulheres no mundo. Em 2007, ocorreram 548 mil óbitos de mulheres pela neoplasia mamária, constituindo-se na quinta causa de mortalidade por câncer ${ }^{1}$. No Brasil, acredita-se que em 2008 tivemos 49.400 casos novos de câncer de mama, com um risco estimado de 51 casos a cada 100 mil mulheres. É o segundo tipo de câncer mais frequente entre as mulheres; o primeiro é o câncer de pele ${ }^{2}$.

$\mathrm{O}$ adoecimento pelo câncer de mama e seu tratamento geram sérias consequências que podem ser temporárias ou permanentes na vida da mulher. A cirurgia mamária, seja ela conservadora ou não, mesmo acompanhada da reconstrução mamária pode ser vivenciada de modo traumático pela mulher, sendo considerada uma mutilação, dependendo da importância dada pela mulher à imagem corporal. Além disso, a funcionalidade do membro superior pode ficar comprometida com o linfedema de braço que surge após a dissecção dos linfonodos axilares. Outro aspecto a ser considerado é a mudança da sensação tátil do seio após sua reconstrução ${ }^{3,4}$.

Os efeitos colaterais advindos da quimioterapia, radioterapia e da hormonioterapia também interferem negativamente no cotidiano, na elaboração da imagem corporal e na vida sexual da mulher. As principais consequencias desse tratamento são náuseas, vômitos, fadiga, disfunção cognitiva, alopecia, ganho de peso, palidez, menopausa induzida, diminuição da lubrificação vaginal e excitação, redução do desejo sexual, dispaurenia e anorgasmia ${ }^{3}$.

O impacto ocasionado pelo câncer na vida da pessoa é enorme, pois além da dor e do desconforto decorrentes da doença e seu tratamento, ocorrem mudanças de ordem psíquica, social e econômica. As perdas econômicas relacionamse aos custos diretos com a compra de medicamentos, busca por procedimentos hospitalares, por outros serviços de saúde, além de custos indiretos como o potencial produtivo perdido ${ }^{5}$.

Mulheres com o câncer de mama passam por reflexões e questionamentos sobre a vida pregressa e futura à doença que afetarão diretamente seu modo de vida e seu comportamento em relação à própria saúde. $\mathrm{O}$ processo que decorre do diagnóstico à intervenção cirúrgica e tratamentos adjuvantes fazem com que surjam mudanças acerca do relacionamento com o parceiro sexual e afetivo, familiares e amigos; confrontação de preconceitos e estigmas; revisão de po- sicionamentos identitários adotados da sexualidade; vida sexual; autoimagem e autoestima; o medo da recorrência da doença; e possíveis quadros de ansiedade e depressã $0^{6-9}$.

A elaboração da imagem corporal pelas pessoas pode ser considerada um fenômeno multidimensional, pois envolve aspectos fisiológicos, psicológicos e sociais, que afeta as emoções, pensamentos e o modo de as pessoas relacionaremse com os outros, influenciando intensamente a qualidade de vida delas ${ }^{10}$.

Entendemos que a imagem corporal envolve, além da percepção e dos sentidos, as figurações e representações mentais que a pessoa tem dos outros e de si mesma, além de emoções e ações advindas da experiência do próprio corpo e do contato com a imagem corporal experienciada por outras pessoas; assim, a imagem corporal é uma construção dinâmica e intercambiável ${ }^{11}$.

Atualmente, o aumento de opções e tecnologias empregadas no diagnóstico e no tratamento do câncer de mama contribuiu para maior sobrevida das mulheres. É fundamental o estudo do cotidiano dessas mulheres, sobretudo na sua (re)elaboração da imagem corporal, visando a subsídios à capacitação de profissionais de saúde na promoção da melhoria da qualidade de vida.

Este artigo tem como objetivo compreender como o câncer de mama e seus tratamentos podem participar da (re)elaboração da imagem corporal da mulher acometida por essa doença, a partir da revisão sistemática de artigos científicos que abordam o impacto do câncer de mama e seus tratamentos na aparência física e na elaboração da imagem corporal das mulheres, publicados entre 2004 e 2009, disponíveis nas bases de dados Medline, Lilacs e PsycInfo.

\section{Metodologia}

Para o alcance dos objetivos propostos neste estudo, utilizamos a revisão sistemática de artigos científicos como metodologia, que permite a obtenção de conhecimentos produzidos na área de modo ordenado e sintético, possibilitando ao leitor o acesso a grande diversidade e complexidade de estudos relevantes num espaço reduzido de tempo ${ }^{12}$.

Para a realização da busca bibliográfica, foram escolhidas as bases de dados científicos Medline, Lilacs e PsycInfo por disponibilizarem grande conteúdo de pesquisas em oncologia em distintas disciplinas da saúde. Foram consultadas diversas combinações de descritores de modo a 
abranger mais artigos que contemplassem especificamente a imagem corporal de mulheres com câncer de mama. Deste modo, foram usadas as seguintes combinações de descritores: body image and breast neoplasms e "imagem corporal e neoplasias da mama”.

As bases de dados científicos foram acessadas durante o mês de julho de 2009, sendo obtidos ao todo 445 textos científicos. Assim, foram obtidos no Medline 351 textos; no Lilacs, foram encontrados cinco; e no PsycInfo, foram alcançados 89 textos. Definimos como critérios de inclusão apenas artigos científicos publicados nos últimos cinco anos (2004 a 2009), nas línguas portuguesa, inglesa, francesa, espanhola ou italiana.

Após a utilização dos critérios de inclusão, houve a redução dos textos a 117 artigos científicos distribuídos em 96 textos do Medline; um artigo do Lilacs e vinte textos do PsycInfo. Além disso, definimos como critério de exclusão a eliminação de artigos cujos resumos não estivessem disponíveis nas bases de dados; textos que abordassem a imagem corporal de mulheres que não tiveram câncer de mama, mas que realizaram a mastectomia profilática; textos que não abordassem a imagem corporal de mulheres com câncer de mama em pelo menos um item dos resultados; e estudos que não pudessem ser obtidos pela internet através do Sistema Integrado de Bibliotecas da Universidade de São Paulo (SIBiNet-USP).

Deste modo, os artigos científicos selecionados foram reduzidos a 63 , com 46 textos obtidos no Medline, um no Lilacs e 13 no PsycInfo, sendo que quatro artigos foram encontrados nas bases de dados Medline e PsycInfo. Assim, o total de artigos utilizados para esta revisão da literatura foram 56, como mostra a Figura 1.

Após a delimitação do número de estudos a serem analisados, foram realizadas a leitura do material e a sistematização dos artigos em categorias. As categorias foram determinadas em razão da similaridade de temas abordados pelos artigos científicos, de modo a oferecer uma discussão dos resultados que permitisse a abordagem de convergências e divergências encontra-

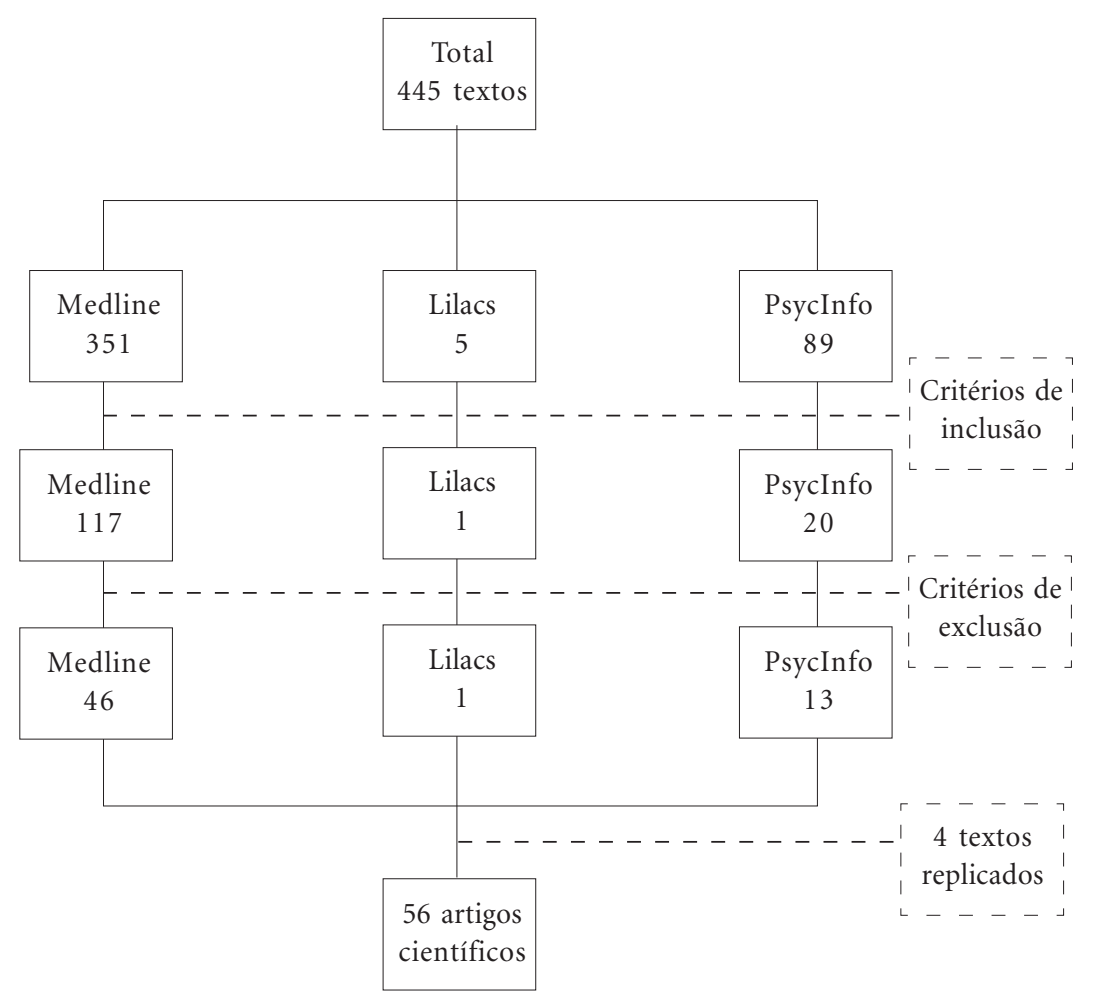

Figura 1. Processo de seleção dos artigos. 
das nos estudos. Também foram obtidas revisões da literatura científica entre os artigos pesquisados; no entanto, optamos por não incluílas nas categorias, e sim citá-las nas considerações finais, de modo a traçar uma comparação dos apontamentos finais dos autores com nossas conclusões.

\section{Resultados}

\section{Mudança da imagem corporal como consequência do tratamento do câncer de mama}

Foram delimitados para esta categoria 18 dos 56 artigos científicos. Tais estudos são importantes para a compreensão do fenômeno analisado, pois o tratamento para o câncer de mama consiste na combinação de diferentes modalidades terapêuticas que geralmente resultam em grandes alterações da aparência da mulher.

A cirurgia mamária constitui-se no principal tratamento, sendo empregada na maioria dos casos. Os procedimentos cirúrgicos recomendados ao tratamento consistem na mastectomia e nas cirurgias conservadoras da mama, como a lumpectomia e a quadrantectomia. Além disso, mulheres que foram submetidas à mastectomia podem ser submetidas à reconstrução imediata da mama ou submeter-se à cirurgia reconstrutora algum tempo depois. A reconstrução da mama também pode ser empregada em mulheres que foram submetidas às cirurgias conservadoras da mama visando corrigir alguma deformidade do seio, quando há a extração de grande parte da mama.

A maioria dos estudos selecionados nesta categoria realizou comparações entre grupos de mulheres que foram submetidas à mastectomia com grupos de mastectomizadas que fizeram a reconstrução mamária e grupos de mulheres que fizeram cirurgias conservadoras da mama, como a lumpectomia. Como era esperado, tais estudos concluem que mulheres submetidas à mastectomia radical sem reconstrução da mama apresentam maiores índices de insatisfação com a própria imagem corporal, além de descontentamento pelo resultado estético da cirurgia, se comparadas às mulheres que fizeram outros procedimentos cirúrgicos para o câncer de mama ${ }^{13-24}$.

O impacto que a cirurgia mamária causa no cotidiano da mulher com câncer de mama foi estudado comparando-se mastectomizadas com outros grupos submetidos a diferentes cirurgias.
As mastectomizadas apresentaram uma frequência maior de problemas com a percepção da imagem corporal. Elas concordaram mais com as assertivas de evitar ir à praia e usar roupas cavadas, além de se isolar mais de amigos do que as mulheres dos outros grupos de comparação ${ }^{23}$.

Mulheres mastectomizadas sem reconstrução mamária tenderam a apresentar maior desejo por uma nova cirurgia estética quando comparadas às mulheres que fizeram cirurgias conservadoras mesmo após um longo período de tempo ${ }^{17}$.

Um estudo japonês comparou grupos de pacientes que foram submetidas à mastectomia poupadora da pele ou à mastectomia poupadora do mamilo, acompanhadas da reconstrução mamária imediata, com mulheres que fizeram somente a mastectomia radical e mulheres que foram submetidas a cirurgias conservadoras da mama. Assim, foram observadas maior satisfação e melhor imagem corporal entre os grupos de reconstrução e de conservação da mama, e menores índices entre o grupo de mastectomizadas radicalmente ${ }^{18}$.

Num estudo que analisou a satisfação da paciente com partes de seu corpo, foi observado que mulheres submetidas à cirurgia conservadora da mama tiveram maior satisfação com a aparência do tórax, enquanto mulheres que fizeram a reconstrução mamária relataram maior satisfação com sua área abdominal ${ }^{23}$. Notamos que tais resultados podem ser devidos ao tipo de cirurgia reconstrutora empregada em que é realizada a extração de tecidos e músculos da área abdominal para implantação na região mamária, gerando um resultado cosmético positivo na área abdominal ao eliminar excessos de pele.

Notamos que em relação ao tipo de cirurgia reconstrutora empregada também foram observados efeitos positivos na imagem corporal, a longo prazo, em grupos de mulheres que fizeram a reconstrução mamária com implante de um extensor anatômico submuscular e grupos com implante submuscular acompanhado do retalho do músculo grande dorsal ${ }^{19}$. A imagem corporal novamente manteve-se positiva em mulheres que fizeram a reconstrução mamária, em um estudo que avaliou grupos de mulheres submetidas às cirurgias de reconstrução com retalho do músculo grande dorsal e do procedimento com expansor tecidual, sendo que alterações da imagem corporal foram observadas somente nas mulheres que tiveram algum tipo de complicação pós-cirúrgica ${ }^{25}$.

Encontramos contradições em relação aos resultados comparativos de alguns estudos. De 
maneira geral, observa-se uma vantagem para as cirurgias conservadoras em relação à reconstrução mamária para os resultados de imagem corporal ${ }^{13,14,21,26}$. Um estudo australiano realizado em 2005 concluiu que mulheres que fizeram a reconstrução mamária apresentaram resultados semelhantes de imagem corporal, mantendo-se elevada em relação aos das pacientes submetidas à cirurgia conservadora da mama ${ }^{20}$.

Observamos que não foram encontradas diferenças nos resultados de imagem corporal quando realizada a comparação de grupos de mulheres submetidas a dois tipos de cirurgia conservadora da mama como a excisão local extensa (wide local excision) via abordagem anterior e a lateral ${ }^{27}$.

A idade foi considerada um fator importante para o ajustamento à aparência alterada pelos tratamentos para o câncer de mama, uma vez que foi observado em alguns estudos que mulheres mais jovens apresentaram maior prejuízo da imagem corporal do que mulheres acima de $50 \operatorname{anos}^{15-17,22,26,28,29}$.

Outra questão refere-se à importância que a mulher atribui à própria aparência física, sendo encontrada maior alteração da imagem corporal em mulheres mais preocupadas com sua aparência física ${ }^{17}$. Tal preocupação foi fator importante para a escolha da cirurgia de reconstrução mamária ou de conservação em mulheres chinesas; já a preocupação com a sobrevivência e a consideração às recomendações médicas foram mais consideradas em mulheres que se submeteram à mastectomia ${ }^{24}$. A tomada de decisão pelo tipo de cirurgia a ser realizada também foi considerada um fator importante para a manutenção de uma imagem corporal positiva pela mulher, pois observamos que mulheres submetidas a cirurgias eleitas por outras pessoas reportaram maior prejuízo da imagem corporal ${ }^{14,16}$.

Além dos estudos que enfocam as consequências da cirurgia mamária para a imagem corporal da mulher, destacamos outros que abordam os demais tratamentos. A quimioterapia, a radioterapia e a hormonioterapia são tratamentos secundários para a maioria dos casos de câncer de mama. Os efeitos da quimioterapia geralmente são vivenciados com angústia e estresse pela mulher em tratamento, uma vez que há uma série de alterações físicas importantes como fadiga, menopausa induzida, ganho ou perda de peso e perda dos cabelos ${ }^{26,29}$.

Notamos, a partir da leitura dos artigos publicados nas bases de dados consultadas, que os estudos que abordam especificamente os trata- mentos adjuvantes ao câncer de mama procuram privilegiar o relato de experiência da mulher que os vivencia, sobretudo a quimioterapia e a radioterapia. Deste modo, optamos por incluir estudos que enfocam tais experiências das mulheres na próxima categoria.

\section{Aspectos psicossociais presentes na (re)elaboração da imagem corporal de mulheres com câncer de mama}

Dentre 56, identificamos 19 artigos científicos que, além de abordar alterações físicas da aparência ocasionadas pelos tratamentos, focaram as demais mudanças de ordem psicossocial que ocorrem no cotidiano da mulher. Tais estudos nos fornecem uma compreensão dos múltiplos aspectos de ordem física, psicológica e social que se fazem presentes na (re)elaboração da sua imagem corporal.

O adoecimento pelo câncer de mama, as modificações físicas provenientes dos tratamentos, o medo da recidiva da doença e o temor da morte foram aspectos considerados propiciadores de mudanças nos modos de mulheres com câncer de mama lidar com o próprio corpo e na relação com as demais pessoas. Alguns estudos trouxeram relatos de mulheres que passaram a fazer questionamentos acerca dos papéis sociais aspirados por elas, como o profissional e materno, a cobrança social para retorno imediato da vida aparentemente normal e o silenciamento do sofrimento após o término do tratamento. Além disso, mulheres manifestaram sentimentos de menor valia e de inutilidade relacionados a efeitos colaterais da radioterapia e quimioterapia, como a fadiga, além da limitação do cumprimento de atividades anteriores devido ao linfedema $a^{30-34}$.

Experiências relacionadas às alterações físicas ocasionadas pela remoção de um seio ou de parte dele, tais como a manutenção da sensação corporal da região que foi removida, a dor fantasma e a assimetria corporal, percebida visualmente e pelo desequilíbrio na distribuição de peso corporal, foram relatadas ${ }^{31}$. Mulheres que fizeram a reconstrução mamária afirmaram não ter reconhecido o seio reconstituído como parte integrante de seu corpo, necessitando de um tempo de adaptação, mesmo para aquelas mulheres que realizaram a reconstrução imediata, uma vez que estas relataram sentimentos de anormalidade ante o novo seio ${ }^{32,35}$.

Outros tipos de alterações físicas resultantes da quimioterapia e da radioterapia também foram relatados, como a preocupação com a mu- 
dança da textura da pele após a radioterapia ${ }^{31}$. Alguns estudos destacaram a necessidade de grande maioria das mulheres em camuflar as alterações físicas de modo a preservar sua privacidade no convívio social, ou de restaurar uma aparência próxima a um ideal de beleza e de feminilidade presentes nas sociedades ocidentai ${ }^{30,36}$.

A alopecia foi descrita em alguns estudos, por mulheres participantes, como uma consequência das mais graves do tratamento para o câncer de mama, mesmo se comparada à perda do seio, uma vez que os cabelos estariam relacionados às características identitárias e à segurança emocio$\mathrm{nal}^{33,34,37}$. Algumas mulheres consideraram que a alopecia resultante da quimioterapia poderia denunciar o câncer ocasionando na perda da privacidade no convívio social. Além disso, houve relatos de constrangimento social ocasionado pela assimetria corporal, que foi superado pelo uso de próteses mamárias externas ou pela reconstrução mamária ${ }^{31,33,36}$.

Os cabelos femininos e a simetria corporal são considerados atributos presentes em ideais de feminilidade, de beleza e de saúde que circulam nas sociedades e no cotidiano das mulheres que passam pelo câncer de mama; assim, mulheres apegadas a tais ideais podem ter maior sofrimento e angústia por terem vivenciado alterações corporais. Porém, alguns discursos midiáticos alternativos a esses ideais padronizados e que valorizavam o enfrentamento da mulher ao câncer de mama foram analisados em alguns estudos ${ }^{28,37}$.

O estilo de enfrentamento ao câncer de mama também foi avaliado em um estudo delimitado para esta categoria, sendo observado que mulheres que manifestam um enfrentamento mais otimista tenderam a ter melhor imagem corporal e apresentaram maiores recursos para o ajustamento psicológico à doença e a seus tratamentos ${ }^{38}$, assim como mulheres que apresentavam maior satisfação com o próprio corpo antes do diagnóstico tiveram maior sobrevida em dez anos ${ }^{39}$.

Outra questão a ser considerada refere-se a aspectos da sexualidade que são alterados pelos tratamentos pelo câncer de mama e que estão diretamente relacionados à imagem corporal. Nesse sentido, alguns estudos descreveram uma correlação da imagem corporal com o funcionamento sexual, uma vez que foram reportados resultados de menores pontuações de imagem corporal relacionados a pior funcionamento sexual; o oposto também foi verificado com melhores resultados de imagem corporal associados a um funcionamento sexual positivo ${ }^{40-42}$. Um estudo trouxe relatos de mulheres que percebe- ram a perda do potencial de excitação e de estimulação sexual pela remoção do seio ${ }^{31}$. A imagem corporal e o funcionamento sexual também foram atrelados à percepção da atratividade sexual pelo parceiro ou da qualidade de relacionamento com o parceiro ${ }^{43-46}$. Percebemos pelos estudos abordados nesta categoria que a imagem corporal da mulher submetida aos tratamentos para o câncer de mama é severamente afetada, e que, portanto, é necessário que sejam elaboradas intervenções visando a novas possibilidades de se lidar com o próprio corpo e nas relações com os outros.

\section{Práticas interventivas visando à melhoria da imagem corporal de mulheres com câncer de mama}

Observamos uma diversidade de intervenções que foram testadas em grupos de mulheres com câncer de mama a partir de seis dos 56 artigos científicos delimitados. Tais estudos abrangeram programas de atividades físicas como a dança ${ }^{48}$, práticas psicoterapêuticas grupais e para casais $^{49,50}$, intervenções psicossociais a partir de programas para grupos de mulheres e para casais ${ }^{51,52}$ e tratamentos estéticos não invasivos para mulheres em tratamento para o câncer de mama ${ }^{53}$.

Notamos que os seis artigos alcançaram resultados positivos para a qualidade de vida de mulheres com câncer de mama, sendo que alguns deles tiveram melhores resultados para a manutenção de uma imagem corporal positiva do que outros.

Um estudo norte-americano avaliou um programa de dança com mulheres com câncer de mama a partir da comparação de dois grupos randomizados. Inicialmente, um grupo intervenção participou do programa e um grupo controle foi direcionado a uma lista de espera, cuja participação no programa ocorreu posteriormente à intervenção do primeiro grupo. Foram realizadas avaliações em ambos os grupos em três períodos distintos, sendo a primeira antes das intervenções; a segunda ocorreu ao término da participação do primeiro grupo; e a terceira avaliação ocorreu após a intervenção com o grupo controle. Os autores observaram bons resultados de imagem corporal e de qualidade em ambos os grupos após a intervenção, ou seja, o grupo intervenção apresentou resultados melhores na segunda avaliação, e ambos os grupos tiveram bons resultados na terceira avaliação, sendo que o primeiro grupo manteve resultados positivos de imagem corporal mesmo após um breve período de tempo ${ }^{48}$. 
Outra intervenção visando à melhoria da imagem corporal dessas mulheres consistiu na realização de procedimentos estéticos não invasivos durante o período de internação pré e póscirúrgicos em mulheres francesas ${ }^{53}$. Foi feita a comparação de um grupo intervenção submetido a tratamentos estéticos e com um grupo controle submetido apenas aos cuidados médicos prescritos à situação de cirurgia para o câncer de mama, sendo que os autores do estudo observaram que as mulheres do grupo intervenção obtiveram melhoria da imagem corporal meses após a cirurgia, se comparadas às mulheres que não passaram pela intervenção.

Observamos que os programas de intervenção abordados nos artigos desta categoria foram delineados em módulos, sobretudo os psicoterapêuticos e psicossociais, sendo que cada componente de tais programas teve por objetivo lidar com algum aspecto que comprovadamente afeta o cotidiano das mulheres que tiveram câncer de mama, como a sexualidade, a imagem corporal e o relacionamento com o parceiro, por exemplo.

Os textos que abordaram as intervenções psicoterapêuticas avaliaram os efeitos de uma terapia para casal combinada à terapia sexual ${ }^{50} \mathrm{e}$ os resultados de uma terapia grupal de orientação cognitivo-comportamental ${ }^{49}$, sendo realizada a comparação de grupos que passaram pelas intervenções psicoterapêuticas com grupos controle cujos sujeitos não participaram de intervenção alguma. Em ambos os estudos, foram utilizadas medidas quantitativas de mensuração de aspectos como depressão, ansiedade e imagem corporal, sendo que os autores do estudo de avaliação de uma terapia psicossexual combinada para casais ${ }^{50}$ observaram a melhora de ajustamento à imagem corporal das mulheres que passaram pela intervenção. Já o estudo em que se avaliou a terapia cognitivo-comportamental para grupos de mulheres ${ }^{49}$ relatou que as mulheres avaliadas não obtiveram melhoras na imagem corporal após a intervenção, e consideraram a necessidade de ampliar o tempo utilizado para a abordagem do tema imagem corporal durante a psicoterapia.

Em relação às intervenções psicossociais, foi desenvolvido um estudo de avaliação de um programa de intervenção que visou à melhoria da imagem corporal e autoestima de mulheres com câncer de mama ${ }^{51}$, e um estudo que analisou um programa de promoção de enfrentamento e ajustamento psicológico ao câncer de mama e ginecológico para casais ${ }^{52}$. Observamos que as mu- lheres que participaram do primeiro estudo de intervenção manifestaram melhoras na imagem corporal $^{51}$, porém não foi observada alteração da imagem corporal de mulheres que foram submetidas à intervenção psicossocial juntamente com os seus maridos, mas estas tiveram uma percepção positiva da aceitação do parceiro em relação a sua imagem corporal ${ }^{52}$.

\section{Construção de instrumentos de coleta de dados visando à avaliação da imagem corporal de mulheres com câncer de mama}

Foram delimitados oito artigos dos 56, sendo que alguns estudos presentes nesta categoria abordaram o desenvolvimento de escalas de avaliação da imagem corporal como item principal ou subitem ${ }^{54,55}$, e outros fizeram a adaptação transcultural de escalas que já são utilizadas ${ }^{56,57}$. Um estudo chinês abordou a análise do conceito de autoeficácia como preditor de ajustamento em mulheres que tiveram câncer de mama. O conceito de autoeficácia refere o envolver de crenças pessoais positivas baseadas no julgamento pessoal sobre a disponibilidade de habilidades individuais necessárias para o alcance de resultados bem-sucedidos. O estudo considerou que mulheres que apresentaram níveis mais elevados de autoeficácia apresentaram melhor autoimagem, porém tenderam a subestimar as consequências negativas da cirurgia mamária em sua aparência e sexualidade ${ }^{58}$.

O uso da fotoelicitação como técnica metodológica foi abordada num estudo que investigou mulheres que fizeram quimioterapia. A fotoelicitação consiste em dar uma câmera fotográfica nas mãos do sujeito pesquisado e solicitar que este faça fotografias sobre o assunto pesquisado e pedir que o sujeito-fotógrafo dê sentido à imagem que foi produzida. Os autores do estudo consideraram o instrumento um importante disparador de narrativas das experiências do adoecimento e que permitem ampla participação das pessoas investigadas no processo de pesquisa ${ }^{59}$. Observamos que o desenvolvimento de instrumentos para o estudo da imagem corporal de mulheres com câncer de mama é imprescindível para que haja melhor compreensão do fenômeno estudado e consequente fomentação de práticas mais eficazes de intervenção em saúde.

Outros estudos que foram selecionados para esta categoria descreveram alguns tipos de tratamentos complementares aos usuais e que visavam minimizar consequências físicas de tratamentos para o câncer de mama, como a técnica 
de scalp cooling para evitar a queda dos cabelos durante a quimioterapia, o uso de certos produtos de curativos e assepsia de feridas malignas ${ }^{60,61}$.

A partir da leitura dos textos delimitados para esta categoria, podemos afirmar que há um amplo leque de instrumentos de avaliação da imagem corporal de mulheres com câncer, assim como o desenvolvimento de tratamentos complementares.

\section{Considerações finais}

Os estudos obtidos nesta revisão da literatura nos possibilitaram explicitar alguns aspectos que podem contribuir para a compreensão das repercussões físicas, psicossociais e culturais que o câncer de mama e seus tratamentos podem ocasionar na (re) laboração da imagem corporal da mulher acometida por esta doença. Além disso, destacamos alguns pontos que consideramos importantes contribuições para a produção de novos conhecimentos sobre o assunto.

Em nossa delimitação de artigos, foram obtidas algumas revisões anteriores da literatura que buscaram abordar diversos aspectos que estão presentes na (re)elaboração da imagem corporal de mulheres com câncer de mama, como a relação de aspectos psiquiátricos envolvidos no processo de adoecimento e ajustamento psicológico ${ }^{62}$; a reconstrução mamária após o câncer de mama ${ }^{63}$; a alopecia induzida pela quimioterapia ${ }^{63}$; câncer de mama em mulheres joven ${ }^{64}$ e questões de imagem corporal ${ }^{65}$, sendo que os principais temas relacionados à imagem corporal abordados por tais revisões foram atualizados neste artigo.

Observamos que entre todos os estudos analisados o conceito de imagem corporal foi pouco problematizado, sendo que, algumas vezes, a imagem corporal foi associada à satisfação da mulher com câncer de mama com a aparência física após o tratamento e ao bem-estar psicológico $^{20,25,67}$.

Em outros estudos, a imagem corporal foi considerada um componente da qualidade de vida, sendo que tais artigos utilizaram escalas de mensuração da imagem corporal e/ou de qualidade de vida. Entendemos que tais autores que não definiram um conceito específico de imagem corporal em seus estudos adotaram a definição das escalas utilizadas. Deste modo, o conceito de imagem corporal advindo de uma das escalas mais utilizadas pelos estudos delimitados neste artigo, o Body Image Scale (BIS), associa elementos afetivos, cognitivos e comportamentais que participam da (re)elaboração da imagem corporal de pessoas com câncer ${ }^{13-15,17,19,21,22,26,27}$.

O caráter individualizado da constituição da imagem corporal também foi referido em alguns estudos qualitativos ${ }^{37,68}$, e outros textos abordaram a relação da imagem corporal da mulher com câncer de mama com a sexualidade e a feminilidade $^{14}$.

Os artigos científicos discutidos no presente trabalho abordaram diversos aspectos a respeito das repercussões físicas dos tratamentos do câncer de mama na vida da mulher, como a perda do seio ocasionada pela cirurgia mamária, sendo que vários estudos apontaram a idade avançada como forte fator de ajustamento psicológico. Notamos que uma hipótese a este resultado refere-se ao fato de que com o processo de envelhecimento o ser humano naturalmente vivencia mudanças corporais significativas que ocorrem num processo gradual, e que muitas vezes tais mudanças também estão relacionadas a modificações de expectativas ou de desempenho de papéis sociais.

Desse modo, parte das repercussões físicas dos tratamentos pelo câncer de mama altera expectativas de alguns papéis sociais femininos que já foram alterados na vivência de mulheres mais velhas, mas que para as mulheres jovens pode gerar um imenso impacto, como a interdição da maternidade advinda da menopausa induzida pela quimioterapia, ou a perda do potencial produtivo pelo linfedema, quando há a dissecção de nódulos axilares.

Ao considerarmos que a imagem corporal envolve aspectos fisiológicos, psicológicos e sociais, que afeta emoções, pensamentos e o modo de as pessoas se relacionarem com as outras, devemos lembrar que o linfedema, além de ter um efeito estético, pode comprometer a execução de tarefas cotidianas que exijam força física e movimentos repetitivos, o que pode gerar grande sofrimento para mulher que o vivencia, ao se perceber impossibilitada de desempenhar algumas atividades que lhe traziam status social ou sustento financeiro.

Observamos a escassez de publicações brasileiras a respeito da imagem corporal de mulheres com câncer de mama, havendo a necessidade de elaboração de estudos que enfoquem, sobretudo, a experiência da mulher brasileira com câncer de mama em relação à sua imagem corporal, contemplando aspectos socioculturais específicos.

Ressaltamos a importância de se pesquisarem os diversos contextos socioculturais a que estamos inseridos para que possamos compre- 
ender como alguns símbolos culturais se fazem presentes na dinâmica da elaboração da imagem corporal, sobretudo quando aspectos físicos que estão atrelados a ideais de beleza são modificados devido à doença e seus tratamentos, como no caso do câncer de mama. Além disso, a observância da presença de discursos alternativos aos modelos ideais pode nos fornecer pistas de que tais padrões culturais não são hegemônicos e que há a busca de outras formas de significar o próprio corpo, o que é feminino e o que é belo. Deste modo, eles se fazem imprescindíveis para o fomento de propostas de intervenção com mulheres com câncer de mama.

\section{Colaboradores}

DB Santos participou do delineamento, análise de dados e redação do artigo. EM Vieira, da concepção, do delineamento, análise e revisão crítica.

\section{Referências}

1. World Health Organization (WHO). Cancer. Fact sheet $n^{\circ}$ 297. Geneva: World Health Organization; 2008. [acessado 2009 jul 17]. Disponível em: http:/ /www.who.int/mediacentre/factsheets/fs297/en/ print.html.

2. Instituto Nacional de Câncer (INCA). Coordenação de Prevenção e Vigilância de Câncer: Estimativas 2008: Incidência de Câncer no Brasil. Rio de Janeiro: INCA; 2007.

3. White CA, Body Images in Oncology. In: Cash TF, Pruzinsky T. Body Image: a Handbook of Theory, Research, and Clinical Practice. New York/London: The Guiford Press; 2002. p.379-386.

4. Duarte TP, Andrade AN. Enfrentando a mastectomia: análise dos relatos de mulheres mastectomizadas sobre questões ligadas à sexualidade. Estudos de Psicologia (Natal); 2003; 8(1):155-163.

5. Silva RCF, Hortale V. Cuidados paliativos oncológicos: elementos para o debate de diretrizes nesta área. Cad de Saúde Pública 2006; 22(10):2055-2006.

6. Azevedo RF, Lopes RLM. Experience of breast cancer diagnosis and radical mastectomy: a phenomenology study. Online Brazilian Journal of Nursing [periodico na Internet]. 2006 [acessado 2009 set 15]; 5(1):[cerca de 15p.]. Disponível em: http://www. doaj.org/doaj?func=openurl\&genre $=$ article\&issn $=16764285 \&$ date $=2006 \&$ volume $=5$ \&issue $=1$ \& spage $=$

7. Regis MF, Simões MFS. Diagnóstico de câncer de mama, sentimentos, comportamentos e expectativas de mulheres. Revista Eletrônica de Enfermagem 2005; 7(01):81-86. 
8. Hoga LAK, Santos L. Mastectomia e sua influência sobre a vivência da sexualidade: análise da produção do conhecimento utilizando uma base de dados informatizada. Revista Mineira de Enfermagem 2003; 7(2):145-151.

9. Pereira Sandrine Gonçales, Rosenhein Daniele Portella, Bulhosa Michele Salum, Lunardi Valéria Lerch, Lunardi Filho Wilson Danilo. Vivências de cuidados da mulher mastectomizada: uma pesquisa bibliográfica. Revista Brasileira de Enfermagem 2006; 59(6):791-795.

10. Pruzinsky T, Cash TF. Understanding Body Images: Historical and Contemporary Perspectives. In: Cash TF, Pruzinsky T. Body Image: A Handbook of Theory, Research, and Clinical Practice. New York, London: The Guiford Press; 2002. p. 3-12.

11. Schilder PA. A imagem do corpo: as energias construtivas da psique. 3.ed. São Paulo: Martins Fontes; 1999.

12. Galvão Cristina Maria, Sawada Namie Okino, Trevizan Maria Auxiliadora. Revisão sistemática: recurso que proporciona a incorporação das evidências na prática da enfermagem. Rev. Latino-Am. Enfermagem 2004; 12(3):549-556.

13. Arndt V, C Stegmaier,Ziegler H, Brenner H. Quality of life over 5 years in women with breast cancer after breast-conserving therapy versus mastectomy: a population-based study. Journal of Cancer Research and Clinical Oncology 2008;134(12):1311-1318.

14. Chang JT-C, C-J Chen, Lin Y, Chien Y, Lin C, Gheng A. "Health-Related Quality of Life and Patient Satisfaction After Treatment for Breast Cancer in Northern Taiwan. International Journal of Radiation Oncology ${ }^{*}$ Biolog $y^{*}$ Physics 2007; 69(1):49-53.

15. Gorisek B, Kranjnc P, Krainc AI. Quality of Life and the Effect on Social Status among Slovenian Women after Breast Cancer Treatment. The Journal of International Medical Research 2009; 37(2):557-566.

16. Figueiredo MI, J Cullen, Hwang Y, Rowland JH, Mandelblatt JS. Breast Cancer Treatment in Older Women: Does Getting What You Want Improve Your Long-Term Body Image and Mental Health? J Clin Oncol 2004; 22(19):4002-4009.

17. Bani MR, K Beckmann, Engel J, Lux MP, Rauh C, Eder I, Bani A HA, Breuel C, Bach A, Beckmann MW, Fasching PA.Correlates of the desire for improved cosmetic results after breast-conserving therapy and mastectomy in breast cancer patients. The Breast 2008; 17(6):640-645.

18. Ueda S, Y Tamaki, Yano K, Okishiro N, Yanagisawa T, Imasato M, Shimazu K, Kim SJ, Miyoshi Y, Tanji Y, Taguchi T, Noguchi S. Cosmetic outcome and patient satisfaction after skin-sparing mastectomy for breast cancer with immediate reconstruction of the breast. Surgery 2008; 143(3):414-425.

19. Gui GPH, Kadayaprath G, Tan A, Faliakou EC, Choy C, Ward A, A'Hern R. Long-Term Quality-of-Life Assessment following One-Stage Immediate Breast Reconstruction Using Biodimensional Expander Implants: The Patient's Perspective. Plastic \& Reconstructive Surgery January 2008; 121(1):17-24.

20. Nano MT, P Grantley Gill,Jollas J, Bochner MA, Carter N, Winefield HR. Qualitative assessment of breast reconstruction in a specialist breast unit. ANZ Journal of Surgery 2005; 75(11):445-453.
21. Nano MT, PG Gill, Kollas J, Bochner MA, Malycha P, Winefield HR. Psychological impact and cosmetic outcome of surgical breast cancer strategies. ANZ Journal of Surgery; 2005; 75(11):940-947.

22. Hopwood P, J Haviland,Mills J, Sumo G, Bliss JM. The impact of age and clinical factors on quality of life in early breast cancer: An analysis of 2208 women recruited to the UK START Trial (Standardisation of Breast Radiotherapy Trial). The Breast 2007; 16(3): 241-251.

23. Parker P, A Youssef, Walker, S, Basen-Engquist K, Cohen L, Gritz ER, Wei QX, Robb GL. Short-Term and Long-Term Psychosocial Adjustment and Quality of Life in Women Undergoing Different Surgical Procedures for Breast Cancer. Annals of Surgical Oncology 2007; 14(11):3078-3089.

24. Monteiro-Grillo I, P Marques-Vidal, Jorge M. Psychosocial effect of mastectomy versus conservative surgery in patients with early breast cancer. Clinical and Translational Oncology 2005; 7(11): 499-503.

25. Potter S, HJ Thomson, Greenwood RJ, Hopwood P, Winters ZE. Health-related quality of life assessment after breast reconstruction. British Journal of Surgery 2009; 96(6):613-620.

26. Janz NK, M Mujahid, Lantz PM, Fagerlin A, Salem B, Morrow M, Deapen D, Katz SJ. Population-based study of the relationship of treatment and sociodemographics on quality of life for early stage breast cancer. Quality of Life Research 2005; 14(6):1467-1479.

27. Lee M, M Patel, Cresswell AB, Bentley PG. Body Image Score Following Anterior and Lateral Approaches to Wide Local Excision for Early Breast Cancer. The Breast Journal 2007; 13(3):38-242.

28. Lam WWT, R Fielding, Ho EYY, Chan M, Or A. Surgeon's recommendation, perceived operative efficacy and age dictate treatment choice by Chinese women facing breast cancer surgery. PsychoOncology 2005; 14(7):585-593.

29. Vargens OMC, CM Berterö. Living With Breast Cancer: Its Effect on the Life Situation and the Close Relationship of Women in Brazil. Cancer Nursing 2007; 30(6):471-478.

30. Thomas-MacLean R. Beyond dichotomies of health and illness: life after breast cancer. Nursing Inquiry 2005; 12:200-209.

31. Sanitt JS. Breast reconstruction: a patient's story. The Breast Breast Cancer in Young Women 2006; 15(Suppl.2):S31-S33.

32. Vilhauer RP. A qualitative study of the experiences of women with metastatic breast cancer" Palliative \& Supportive Care 2008; 6(03):249-258.

33. Schnur JB, SC Ouellette, Bovbjerg DH, Montgomery GH. Breast Cancer Patients' Experience of External-Beam Radiotherapy. Qual Health Res 2009; 19(5):668-676.

34. Hill O, K White. Exploring Women's Experiences of TRAM Flap Breast Reconstruction After Mastectomy for Breast Cancer. Oncology Nursing Forum; 2008. 35(1):81-88

35. Frith H, D Harcourt. Anticipating an altered appearance: Women undergoing chemotherapy treatment for breast cancer. European Journal of Oncology Nursing 2007; 11(5):385-391. 
36. Browall M, Gaston-Johansson F, Danielson E. Postmenopausal Women With Breast Cancer: Their Experiences of the Chemotherapy Treatment Period. Cancer Nursing 2006; 29(1):34-42.

37. Ucok O. The fashioned survivor: Institutionalized representations of women with breast cancer. Communication \& Medicine 2007; 4(1):67-78.

38. Ryan C. Am I Not a Woman? The Rhetoric of Breast Cancer Stories in African American Women's Popular Periodicals. Journal of Medical Humanities 2005; 25(2):129-150.

39. Vos PJ, B Garssen, Visser AP, Duivenvoorden HJ, Haes HC. Early Stage Breast Cancer: Explaining Level of Psychosocial Adjustment Using Structural Equation Modeling. Journal of Behavioral Medicine 2004; 27(6):557-580.

40. Cousson-Gelie F, M Bruchon-Schweitzer, Dilhuydy JM, Jutand M. Do Anxiety, Body Image, Social Support and Coping Strategies Predict Survival in Breast Cancer? A Ten-Year Follow-Up Study. Psychosomatics 2007; 48(3):211-216.

41. Ramos AS, I Patrão. Imagem corporal da mulher com cancro de mama: Impacto na qualidade do relacionamento conjugal e na satisfação sexual. Análise Psicológica 2005; 23(3):295-304.

42. Garrusi B, H Faezee. How do Iranian women with breast cancer conceptualize sex and body image? Sexuality and Disability 2008; 26(3):159-165.

43. Speer JJ, Hillenberg B, Sugrue DP, Blacker C, Kreuge CL, Decker VB, Zakalik D, Decker DA. Study of Sexual Functioning Determinants in Breast Cancer Survivors. Breast Journal 2005; 11(6):440-447.

44. Adachi K, T Ueno, Fuijoka T, Fujitomi Y, Ueo H. Psychosocial Factors Affecting the Therapeutic Decision-making and Postoperative Mood States in Japanese Breast Cancer Patients who underwent Various Types of Surgery: Body Image and Sexuality. Jpn. J. Clin. Oncol. 2007; 37(6):412-418.

45. Takahashi M, I Kai. Sexuality after breast cancer treatment: Changes and coping strategies among Japanese survivors. Social Science \& Medicine 2005; 61(6):1278-1290

46. Sheppard LA, Ely S. Breast Cancer and Sexuality. The Breast Journal, Australia 2008; 14(2):176-181.

47. Fobair P, SLS Subo Chang, C D’Onofrio, PJ Banks, JR Bloom. Body image and sexual problems in young women with breast cancer. Psycho-Oncology 2006; 15(7):579-594.

48. Sandel SL, Judge JO, Landry N, Faria I, Ouellete R, Majczak M. Dance and Movement Program Improves Quality-of-Life Measures in Breast Cancer Survivors. Cancer Nursing 2005; 28(4):301-309.

49. Narvárez A, C Rubiños, Corté-Funes F, Gómes R, Garcia A. Valoración de la eficacia de una terapia grupal cognitivo-conductual en la imagen corporal, autoestima, sexualidad y malestar emocional (ansiedad y depresión) en pacientes de cáncer de mama. Psicooncología 2008; 5(1):93-102.

50. Kalaitzi C, VP Papadopoulos, Michas K, Vlasis K, Skandalakis P, Filipou D. Combined brief psychosexual intervention after mastectomy: Effects on sexuality, body image, and psychological well-being. Journal of Surgical Oncology 2007; 96(3):235-240.
51. Sebastián J, D Manos, Bueno MJ, Mateos N. Imagen corporal y autoestima en mujeres con cáncer de mama participates en un programa de intervención psicosocial. Cliníca y Salud 2007; 18(2):137-161.

52. Scott JL, WK Halford, Ward B. United We Stand? The Effects of a Couple-Coping Intervention on Adjustment to Early Stage Breast or Gynecological Cancer. Journal of Consulting and Clinical Psychology 2004; 72(6):1122-1135.

53. Quintard B, F Lakdja. Assessing the effect of beauty treatments on psychological distress, body image, and coping: A longitudinal study of patients undergoing surgical procedures for breast cancer. Psycho-Oncology 2008; 17(10):1032-1038.

54. Baxter NN, PJ Goodwin, Mcleod RS, Dion R, Devins G, Bombardier C. Reliability and Validity of the Body Image after Breast Cancer Questionnaire. The Breast Journal 2006; 12:221-232.

55. Erci B, N Karabulut. Appraising the self-assessed support needs of Turkish women with breast cancer. European Journal of Cancer Care 2007; 16:137143.

56. Erci B. Psychometric evaluation of Self-Assessed Support Needs of women with breast cancer Scale. Journal of Clinical Nursing 2007; 16:1927-1935.

57. Brédart A, AS Verdier, Dolbeault S. Traduction/adaptation française de l'échelle. Body Image Scale (BIS) évaluant la perception de l'image du corps chez des femmes atteintes de cancer du sein. PsychoOncologie 2007; 1(1):24-30.

58. Lam WWT, R Fielding. Is self-efficacy a predictor of short-term post-surgical adjustment among Chinese women with breast cancer? Psycho-Oncology 2007; 16(7): 651-659.

59. Frith H, D Harcourt. Using Photographs to Capture Women's Experiences of Chemotherapy: Reflecting on the Method. Qual Health Res 2007; 17(10):13401350.

60. Mols F, CJ van den Hurk, Vingerhoets A, Breed WPM. Scalp cooling to prevent chemotherapy-induced hair loss: practical and clinical considerations. Supportive Care in Cancer 2009;17(2): 181-189.

61. Lund-Nielsen B, Müller K, Adamsen L. Malignant wounds in women with breast cancer: feminine and sexual perspectives. Journal of Clinical Nursing 2005; 14:56-64.

62. Cantinelli FS, Camacho RS, Smaletz O, Gonsales BK, Braguittoni E, Rennó Jr J. A oncopsiquiatria no câncer de mama: considerações a respeito de questões do feminino. Rev. Psiquiatr. Clín. 2006; 33(3):124133.

63. Ceradini, DJ, JP Levine . Breast cancer reconstruction: More than skin deep. Primary Psychiatry 2008; 15(10):72-80.

64. Brennan M, FJ Houssami N, Kirk J, Boyages J. Breast cancer in young women. Aust Fam Physician 2005; 34(10):851-855.

65. Lemieux J, Maunsell E, Provencher L. Chemotherapy-induced alopecia and effects on quality of life among women with breast cancer: a literature review. Psycho-Oncology 2008;17(4):317-328.

66. Helms RL, O'Hea E, Corso M. Body image issues in women with breast cancer. Psychology, Health o Medicine 2008; 13(3):313-325. 
67. Wang, HT, CM Barone, Steigelman MB, Kahlenberg M, Rousseau D, Berger J, Daum A, Ortegon DP. Aesthetic outcomes in breast conservation therapy. Aesthetic Surg J 2008; 28(2):165-170.

68. Boehmke MMRNDNSA, SSRNDNS Dickerson. Symptom, Symptom Experiences, and Symptom Distress Encountered by Women With Breast Cancer Undergoing Current Treatment Modalities. Cancer Nursing 2005; 28(5):382-389.

Artigo apresentado em 18/09/2009

Aprovado em 17/01/2010

Versão final apresentada em 18/02/2010 